\title{
Confinement Effects on the Crystalline Features of Poly(9,9-dioctylfluorene)
}

Jaime Martin ${ }^{1, *}$, Alberto Scaccabarozzi ${ }^{1}$, Aurora Nogales ${ }^{2}$, Ruipeng Li $^{3}$, Detlef-M. Smilgies ${ }^{3}$, Natalie Stingelin ${ }^{1, *}$

${ }^{1}$ Centre for Plastic Electronics and Department of Materials, Imperial College London, Exhibition Road, London, SW7 2AZ

${ }^{2}$ Instituto de Estructura de la Materia IEM-CSIC, C/ Serrano 121, Madrid 28006, Spain

${ }^{3}$ Cornell High Energy Synchrotron Source, Wilson Laboratory, Cornell University, Ithaca, New York 14853, USA

\begin{abstract}
Typical device architectures in polymer-based optoelectronic devices, such as field effect transistors organic light emitting diodes and photovoltaic cells include sub-100 nm semiconducting polymer thin-film active layers, whose microstructure is likely to be subject to finite-size effects. The aim of this study was to investigate effect of the two-dimensional spatial confinement on the internal structure of the semiconducting polymer poly(9,9-dioctylfluorene) (PFO). PFO melts were confined inside the cylindrical nanopores of anodic aluminium oxide (AAO) templates and crystallized via two crystallization strategies, namely, in the presence or in the absence of a surface bulk reservoir located at the template surface. We show that highly textured semiconducting nanowires with tuneable crystal orientation can be thus produced. The results presented here demonstrate the simple fabrication and crystal engineering of ordered arrays of PFO nanowires; a system with potential applications in devices where anisotropic optical properties are required, such as polarized electroluminescence, waveguiding, optical switching, lasing, etc.
\end{abstract}




\section{Introduction}

Understanding how the microstructure of polymers develops in spatial confinement continues to be a major fundamental issue in the field of soft condensed matter [1-3]. With the rapid rise of nanotechnologies, elements and devices based on polymeric components organized at the sub-micron scale begin to play an important role in a variety of fields, such as photovoltaics, lighting, tissue engineering, sensing, information storage, and more [4, 5]. Hence, the understanding and manipulation of the crystalline features of nanoconfined polymer materials emerge as a major issue for the nanodevice optimization. Note that the mechanical, optical, chemical and/or electrical properties of the device are directly correlated with the crystallinity, crystalline phase, size of crystals, orientation, defects, etc.

Active components of polymer-based optoelectronic devices are clear examples of functional systems where the polymer material -a semiconducting polymer- is processed into nanoscale architectures. Thus, typical device geometries in polymeric field effect transistors, organic light emitting diodes and organic photovoltaic cells include sub-100 nm semiconducting polymer thin-film active layers $[6,7]$, whose microstructure is likely to be subject to finite-size effects. However, little attention has been hitherto paid to the consequences of the spatial limitation on the structural development in semiconducting polymer nanostructures, even though it is well accepted that the microstructure determines to a large extent the device performance[8].

In the last years, the system composed of polymers confined into anodic aluminum oxide (AAO) nanopore arrays has stood out as an efficient tool to study the impact of nanoscale confinement on the crystallization of commodity polymers [9-12]. AAO nanopores constitute an ideal confining medium to assess this issue, as the degree of confinement can be easily tuned by varying the pore diameters from 10 to $400 \mathrm{~nm}[13,14]$. Moreover, due to the well-defined cylindrical pores with rigid walls, the polymer melt can be considered to be effectively confined in a two-dimensional geometry. This is a significant advantage compared to templates like controlled porous glasses (CPG) having interconnected tortuous channels, or cylinder forming block copolymers, in which crystallization often dominates over microphase separation, so that the nanoscopic domain structure does not efficiently confine the crystallizing component. Furthermore, pores in AAO are arranged into a well-aligned hexagonal array, which permits investigating orientation.

Several studies have been published which include basic structural characterization of semiconducting polymer nanostructures prepared from AAO templates [15-18]. Nevertheless, 
most of these have focused on low aspect-ratio (length to diameter) nanostructures, whose crystalline features are known to be largely influenced by the supporting substrate. Recently, the internal structure of a semiconducting polymer (poly(3-hexylthiophene, P3HT) crystallized from the melt inside high aspect-ratio AAO nanopores has been reported [19]. This study revealed a strong impact of the $2 \mathrm{D}$-confinement on the structure development of P3HT. For instance, the stabilization of an uncommon polymorph, i.e. the form II, was observed under strong confinement conditions, as imposed by 15 and $25 \mathrm{~nm}$ in diameter pores. Furthermore, it was found that the crystal texture of the nanowires varied as a function of the degree of confinement (pore diameter): In large diameter pores (above $100 \mathrm{~nm}$ ), crystals were oriented laying the $\pi-\pi$ stacking direction parallel to the long axis of the nanopores, as corresponding to texture governed by the kinetics selection rules of the crystal growth process. Conversely, inside sub-100 nm pores, the strong confinement led to nanowires in which the $\pi-\pi$ stacking direction pointed normal to their longitudinal axis. Along the same line, it is worth noting the work by O'Carroll et al., which showed that the poly(9,9-dioctylfluorene) (PFO) melt crystallized aligning polymer chains with the pore axis when confined in commercial AAO filters (200 $\mathrm{nm}$ in nominal pore diameter) [20]. Such orientation is uncommon for polymers confined in nanopores -it has just been observed for PFO, poly(ethylene oxide, PEO) and syndiotactic polystyrene (sPS) [21, 22],- and its origin is not well understood yet. However, it provokes highly desired anisotropic optical properties in the nanowires in conjugated polymers [23]. Thus, these last two works suggest that semiconducting polymers in confinement may behave different compared to commodity polymers, which highlights that more work is needed to elucidate how the microstructure of these materials is developed under spatial restriction.

Hence, we set out to address the effect of 2D-confinement on the internal structure of the semiconducting polymer PFO (Figure 1a), with the ultimate aim to predict and manipulate the crystalline features of this semiconductor. For that, we have investigated PFO nanowires produced via two processing routes: i) First, the crystallization of confined PFO was carried out at low cooling rate while connected by a surface bulk reservoir located at the template surface (Figure 1b). ii) Second, the confined PFO was crystallized al relatively high cooling rates while isolated in each nanopore (in the absence of the surface film reservoir, Figure 1c). Thus, we show that we are able to (i) fabricate highly texturized semiconducting nanowires and to (ii) manipulate their crystal orientation. The results presented here provide a convincing demonstration of the simple fabrication and crystal engineering of ordered arrays of PFO nanowires; a system with a clear potential for applications where anisotropic optical properties 
are needed, such as polarized electroluminescence, waveguiding, optical switching, lasing, and more $[4,23,24]$.
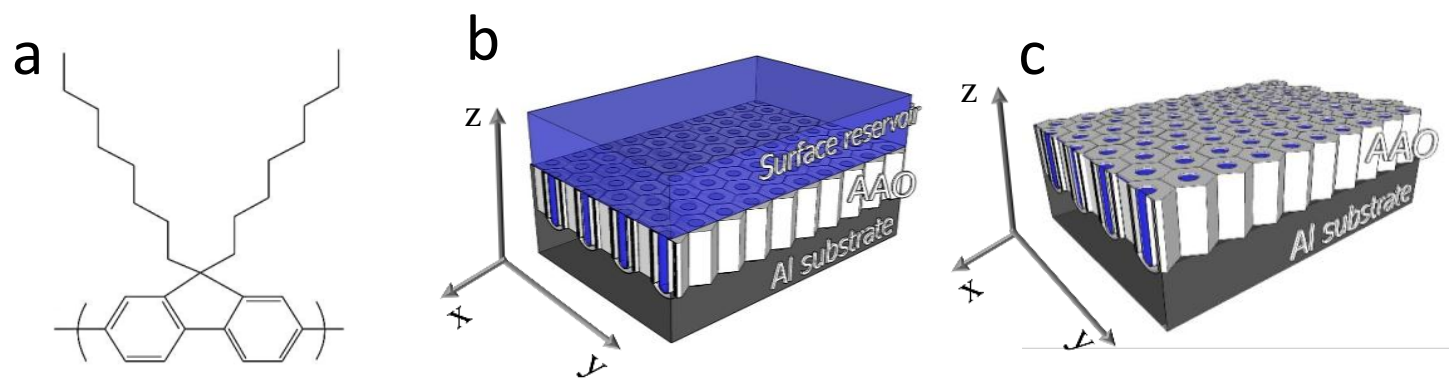

Figure 1. (a) Chemical structure of poly(9,9-di-n-octyl-2,7-fluorene) (PFO). Illustrations of an AAO template containing PFO nanowires connected by a macroscopic polymer reservoir (b) and an AAO template containing PFO nanowires isolated in each nanopore (c). The AAO templates have been coloured in white, while the PFO appears in blue. Axis indicating analysed directions are also included.

\section{Experimental}

Materials. Bulk PFO was purchased from American Dye Source, Quebec, Canada (cat. No. ADS129BE), and used as received. The chemical structure of PFO is illustrated in Figure 1a. The weight-averaged molecular weight, $\overline{M_{w}}$, of the polymers used in this work is $45,000 \mathrm{~g} / \mathrm{mol}$ according to specifications. However, it was estimated to be $24,000 \mathrm{~g} / \mathrm{mol}$ by Chen et al. [25] based on light scattering results of Grell et al. [26]. The AAO templates were purchased from Smart Membranes GMbH, Halle, Germany, and were treated as indicated below.

Infiltration of PFO into the Nanopores. The AAO templates were first sonicated in different polarity solvents (hexane, acetone, isopropanol and water), in order to remove organic molecules attached to the pore walls, which would decrease the surface energy of the walls. For the infiltration, solid PFO pieces were placed on the template surface and annealed at 200 ${ }^{\circ} \mathrm{C}$ for $4 \mathrm{~h}$ under vacuum. Due to the large surface energy difference between the hydroxilated pore walls of the AAO template and the PFO melt, the polymer spontaneously wets the pore walls, which triggers the complete infiltration of the molten PFO in the [27]. One set of samples were non-isothermally crystallized at a cooling rate of $-0.5^{\circ} \mathrm{C} / \mathrm{min}$ while the PFO contained inside the pores was in contact with a macroscoscopic drop of molten PFO that remained on the template surface (on the following denoted as surface bulk reservoir). For the analysis, the residual bulk surface reservoir was removed from the template surface with a sharp razor blade 
and a paper tissue slightly damp with toluene. A second set of samples was rapidly quenched in ice-water immediately after the infiltration process. Subsequently, the surface bulk reservoir was removed as indicated above and, finally, the samples were annealed at $200{ }^{\circ} \mathrm{C}$ for $10 \mathrm{~min}$ and crystallized at $50{ }^{\circ} \mathrm{C} / \mathrm{min}$.

Characterization. The morphology of the AAO templates was characterized by scanning electron microscopy (SEM, Hitachi SU8000).

Wide-angle X-ray scattering measurements were performed at D-line, Cornell High Energy Synchrotron Source (CHESS) at Cornell University. A wide band-pass (1.47\%) X-ray beam with a wavelength of $1.155 \AA$ was shone on the samples with incidence angles between $0.5^{\circ}$ and $1^{\circ}$. A Pilatus $200 \mathrm{k}$ detector with a pixel size of $172 \mu \mathrm{m}$ was placed at a distance of $28.9 \mathrm{~cm}$ from the samples. A $1.5 \mathrm{~mm}$ wide tantalum rod was used to block the intense scattering in the small-angle area. The exposure time for room temperature measurements was $1 \mathrm{~s}$. The nanowire arrays were placed in the setup so that the PFO nanowires were aligned with the $z$-direction (Figures $1 \mathrm{~b}$ and 1c). For the temperature resolved WAXS measurements, a hot stage was employed and heating and cooling rates of approx. $50{ }^{\circ} \mathrm{C} / \mathrm{min}$ were used.

Additional X-ray diffraction $\theta / 2 \theta$ scans were performed to at room temperature using a PANalytical X'Pert Pro MPD employing the $\mathrm{Cu} \mathrm{K} \alpha$ radiation. The analysed wave vector $q$ was parallel to the long axis of nanowires.

Differential scanning calorimetry (DSC) was performed on a Mettler-Toledo DSC 1 Stare system at a heating/cooling rate of $20^{\circ} \mathrm{C} / \mathrm{min}$.

\section{Results and discussion}
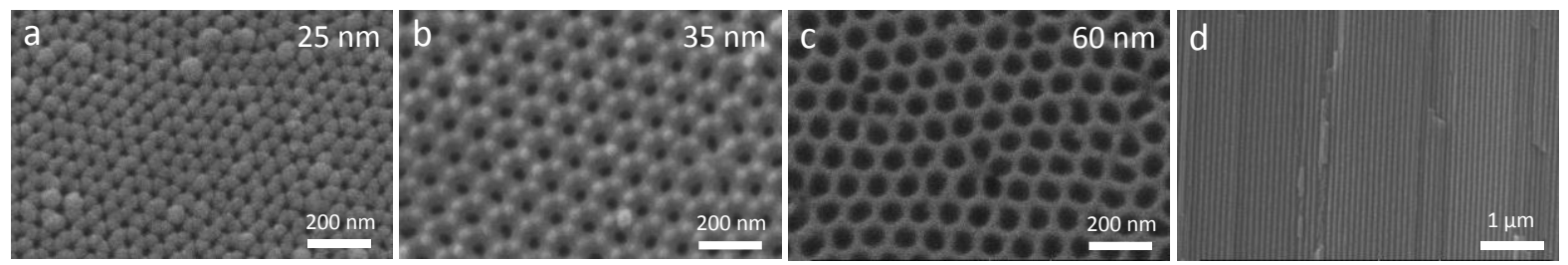

Figure 2. SEM images of the surface of AAO templates having 25 (a), 35 (b) and 60 (c) nm in diameter pores.

(d) Cross section view of the AAO template showing the perfectly aligned array of nanopores. 
The prepared samples consisted of hexagonal arrays of 25, 35, and $60 \mathrm{~nm}$ in diameter PFO nanowires embedded into AAO templates. The birds-eye-views and cross section of the used templates are shown in Figure 2. Thus, we explore the influence of the crystallization strategies applied on the crystalline features of the confined PFO.

\subsection{Crystallization of the PFO nanowires in contact with the bulk surface bulk reservoir} at low cooling rate
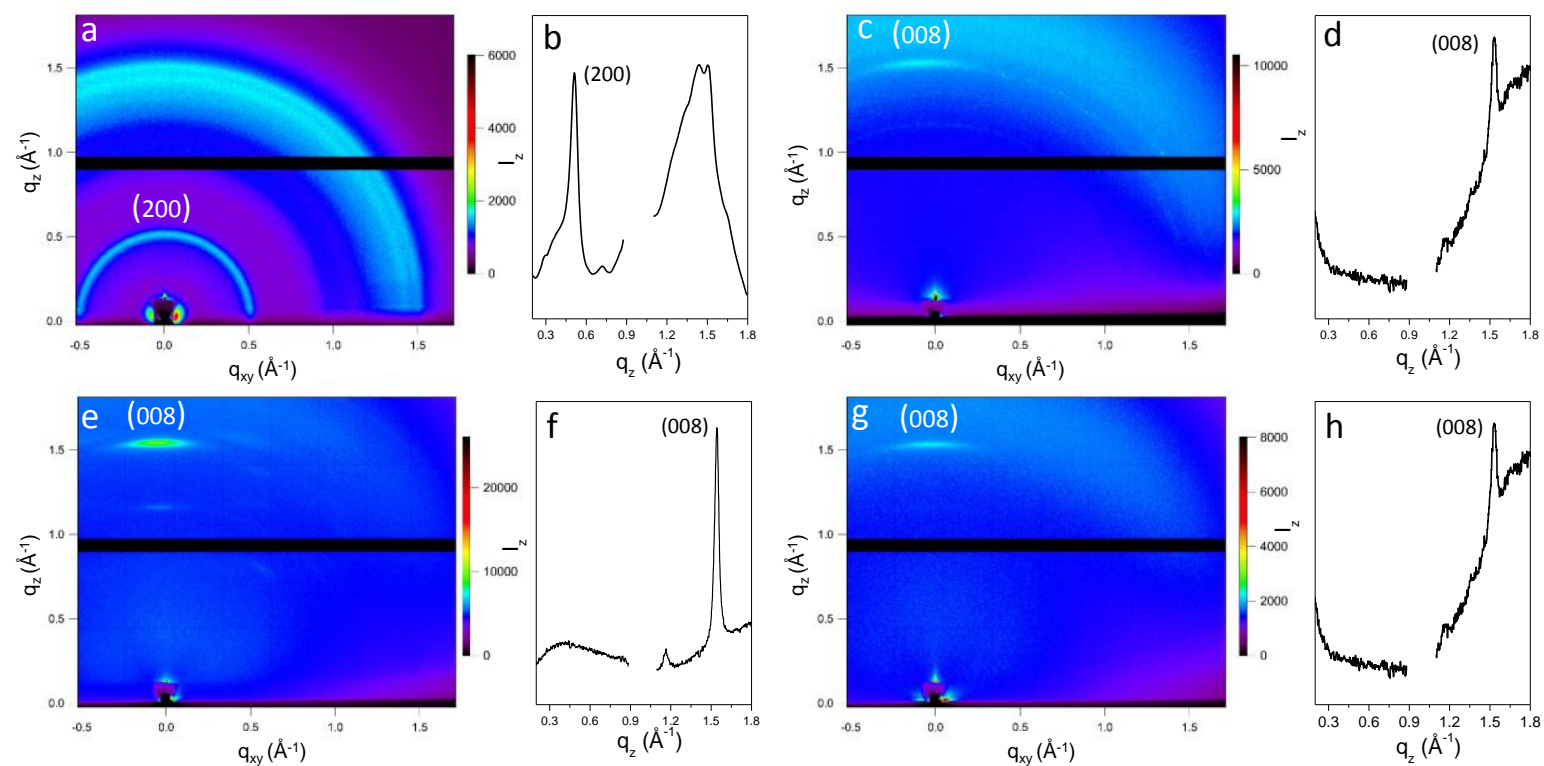

Figure 3. 2D-WAXS patterns of bulk PFO (a) and PFO confined inside $60 \mathrm{~nm}$ (c), $35 \mathrm{~nm}(\mathrm{e})$, and $25 \mathrm{~nm}(\mathrm{~g})$ in diameter pores crystallized at $0.5^{\circ} \mathrm{C} / \mathrm{min}$ in the presence of the surface reservoir. (b, $\mathrm{d}, \mathrm{f}$, and $\mathrm{h}$ ) correspond to the intensity collected along the $z$-direction (parallel to the long-axis of the nanopores) for the bulk, $60 \mathrm{~nm}, 35$ $\mathrm{nm}$, and $25 \mathrm{~nm}$, respectively.

Figure 3 shows the 2D-WAXS patterns of bulk PFO (Figure 3a), and PFO confined inside 60 $\mathrm{nm}$ (Figure 3c), $35 \mathrm{~nm}$ (Figure 3e), and $25 \mathrm{~nm}$ (Figure 3g) pores (in diameter) crystallized with the bulk surface reservoir at $-0.5^{\circ} \mathrm{C} / \mathrm{min}$. All the samples, including the nanowires, show clear Bragg reflections, which indicates a semicrystalline nature of PFO, despite of being crystallized under strong confinement conditions. This characteristic is well-illustrated by the $2 \mathrm{D}$-pattern of the $35 \mathrm{~nm}$ in diameter PFO nanowires, which shows multiple, narrow reflections in a fibrepattern-like diffractogram. In contrast, bulk PFO shows diffraction rings, corresponding to an isotropic distribution of crystals. Intensity along the meridian (z-direction) reveals a 
diffractogram characterised by a prominent diffraction maximum at $q=0.51 \AA^{-1}$, large amount of diffuse scattering and few Bragg reflections. According to Chen et al. [25], such a diffractogram can be ascribed to $\alpha^{\prime}$ phase crystals of PFO. They described the $\alpha^{\prime}$ phase as a kinetically favoured modification of the orthorhombic $\alpha$ form [25]. However, its metastability and high degree of disorder resemble closely a solid state mesophase rather than a real crystalline structure. Such kinds of polymeric solid state mesophases are extremely frequent and are typically characterized by long range order in the parallel arrangement of chain axes like the one observed for PFO (represented by the (200) peak at $q=0.51 \AA^{-1}$ ) and a large amount of structural disorder [28]. For example, mesophases have been identified in poly(ethylene terephthalate) (PET)[29], syndiotactic polystyrene (sPS) [30] or in quenched isotactic and syndiotactic polypropylene[31]. In fact, the PFO itself possesses a well know solid state mesophase, i.e. the $\beta$ phase [32, 33].

Conversely, WAXS patterns of the PFO nanowires were characterized by the presence of well defined, discrete reflections that indicate high degree of orientation of PFO crystals inside the nanopores. Independently on the pore diameter, the diffractograms are characterised by a single diffraction maximum along the $z$-direction, which is parallel to the long axis of the nanopores (Figures 2d, 2f, 2g) (see also Figure 1b). These reflections can be assigned to the $\alpha$ phase using the indexing given by Chen et al. [34]. The peak is centred at $q=1.54 \AA^{-1}$ ( $d$-spacing of 0.416 $\mathrm{nm}$ ) and can be identified as the stacking of (008) lattice planes with a $d$-spacing of $0.415 \mathrm{~nm}$; commensurate with the interphenylene spacing along the aromatic backbone (i.e., half of the repeating unit of the polymer). Such a diffractogram indicates that the polymer chains in the crystal (the $c$-axis) lay along the direction of the long axis of the nanopores.
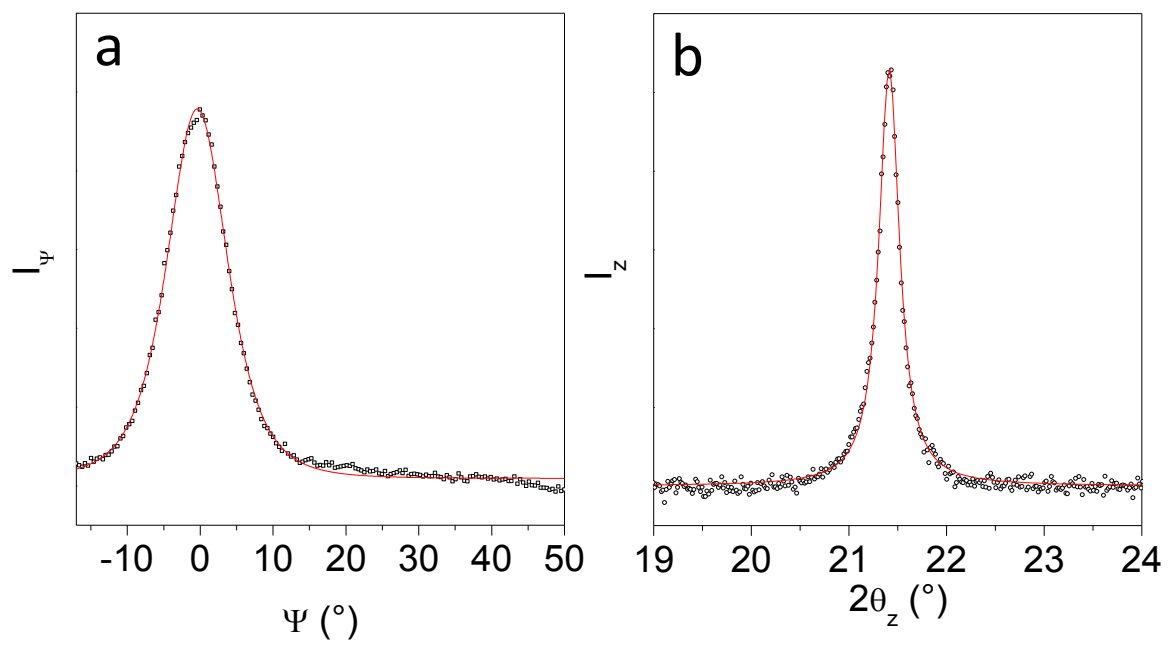
Figure 4. (a) WAXS intensity of the (008) peak of the ensemble of $35 \mathrm{~nm}$ in diameter PFO nanowires along the $\Psi$ angle (defined as the angle between the $\mathrm{z}$ direction and the $\mathrm{x}-\mathrm{y}$ plane). (b) Detail of the (008) peak of the 35 $\mathrm{nm}$ in diameter PFO nanowires as measured in $\theta-2 \theta$ configuration (black dotted line). Corresponding fits to Lorentzian functions are shown as red solid lines.

The orientational distribution of crystals along the $\Psi$ angle -defined as the angle produced by the rotation of the sample normal from the $z$ direction to the $x$ - $y$ plane- was evaluated from the 2D-WAXS intensity at $q=1.54 \AA^{-1}$, i.e. the (008) reflection. Figure 4a depicts the intensity collected (black squares) for the $35 \mathrm{~nm}$ in diameter nanowires together with the fit of the experimental data to a Lorentzian function (red line). This sample was deliberately selected due to its high crystallinity (as deduced from the WAXS results in Figure 3e), but this behaviour is representative of the whole set of samples. The plot is characterized by a maximum at $\Psi=0^{\circ}$-i.e. the $z$-direction and the pore long axis direction- and a full-with-at-the-halfmaximum (FWHM) of $9.7^{\circ}$, which clearly indicate that polymer chains are well aligned, being axially oriented along the nanopores. Indeed, such a low value of FWHM is usually consistent with an exclusive crystal orientation [35].

It must be pointed out here that the texture observed in our nanowires is rather uncommon for polymers crystallized inside cylindrical domains; especially when these are crystallized under conditions promoting low density of nucleation centres, like in the present work (crystallization was induced at $-0.5^{\circ} \mathrm{C} / \mathrm{min}$ ). Within cylindrical nanopores, polymers tend to crystallize along one or several $[h k l]$ directions with zero $l$-index, which typically lay parallel to the long axis of the pore $[9-11,35]$. The $l$-index is usually associated with the $c$-axis of the crystal, which is parallel to the polymer chain direction in the unit cell by convention. Thus, crystals typically grow along the pores with the polymer chains perpendicularly to the pore long axis. The main reason for this behaviour is that the alignment of the chains normal to the pore axis allows lamellae to propagate straight on the pore.

Furthermore, when polymer melts are crystallized inside AAO pores in the presence of a surface reservoir at low cooling rates, the polymer nanowires frequently exhibit uniaxial crystal orientation where the crystallographic direction having the fastest growth rate - it fulfils the zero $l$-index- orients parallel to the pore axis- lays parallel to pore axis. For most of $\pi$ conjugated polymers, including PFO, the fastest growth direction is close to the direction of the $\pi-\pi$ stacking[36]. Hence, one would have expected to observe PFO crystals with the $\pi-\pi$ 
stacking direction parallel to the pore axis. In contrast, our nanowires crystallized with the polymer chains parallel to the pore axis.

In order to gain information about the length-scale of such anomalous orientation, the coherence length of the (008) reflection was calculated[37]. Since the (008) lattice plane is normal to the chain direction, its coherence length is related to the crystal size along such direction and, therefore, it can be related to the thickness of the lamellae. For the analysis, the ensemble of $35 \mathrm{~nm}$ in diameter PFO nanowires was measured in $\theta-2 \theta$ configuration in such a way that the analysed scattering vector, $q$, was parallel to the pore axis $\left(q=q_{z}\right)$ (Figure $\left.4 \mathrm{~b}\right)$. Note that although the experimental broadening was neglected, the resolution in $q$ is better than in the GIWAXS setup used for the texture analysis. The measured FWHM accounted to $0.25^{\circ}$ corresponding to a coherence length of $23 \mathrm{~nm}$ along the aromatic chain direction. Since the length of the repeating unit in PFO is $0.83 \mathrm{~nm}$, one can roughly estimate that the crystals possess 28 repeating units aligned in the pore direction. Such value lays between the numberaverage degree of polymerization $\left(\overline{X_{n}}=24\right)$ and its weight-averaged counterpart $\overline{X_{w}}(=62)$ reported by Chen et al [25], which would suggest that a significant fraction of the crystals may consist of entire molecules. That is, PFO nanowires crystallized with bulk surface reservoir may be composed of extended-chain crystals. However, this must be considered just a speculation, as we lack experimental data on the molecular size of the PFO employed here and the Scherrer equation must be considered just an approximate measurement of the crystallite size. Unfortunately, the (008) reflection was not sufficiently well resolved for bulk PFO, which prevented the comparison between the samples.

On account of such large coherence length of the confined crystals along the aromatic chain direction, we tried to further assess the lamellar thickness in our PFO nanowires by small angle X-ray scattering (SAXS). However, the data collected by GISAXS were not conclusive in part due to the poor contrast in this material (results not shown). Likewise, we tried to assess the thickness of lamellae by analysing the melting temperatures of crystals $\left(T_{m}\right)$ by DSC. According to the Gibbs-Thomson relation [38] (equation 1), small variations of the lamellar thickness lead to crystals with significantly different melting temperatures [64].

$$
\Delta T_{m}=T_{m}^{0}-T_{m}=\frac{2 T_{m}^{0}}{\rho \Delta H_{f, m}}\left(\frac{\gamma_{1}}{L_{1}}+\frac{\gamma_{2}}{L_{2}}+\frac{\gamma_{3}}{L_{3}}\right)
$$


$T_{m}^{0}$ is the melting temperature of an infinitely large crystal, $\rho$ is the crystal density, $\Delta H_{f, m}$ is the heat of fusion per unit of mass, $\gamma_{1}, \gamma_{2}$, and $\gamma_{3}$ are the surface energies of the crystal faces, and $L_{1}, L_{2}$, and $L_{3}$ are the thickness, width, and length of the lamellae, respectively. Given that lateral dimensions of bulk polymer crystals can be considered infinite, the second and the third term of the bracket in equation (1) can be typically neglected [39]; which is why the melting point depression of bulk polymers is generally related just to the reciprocal lamellar thickness $\left(L_{1}^{-1}\right)$ and the free energy of the fold surfaces $\left(\gamma_{1}\right)$.
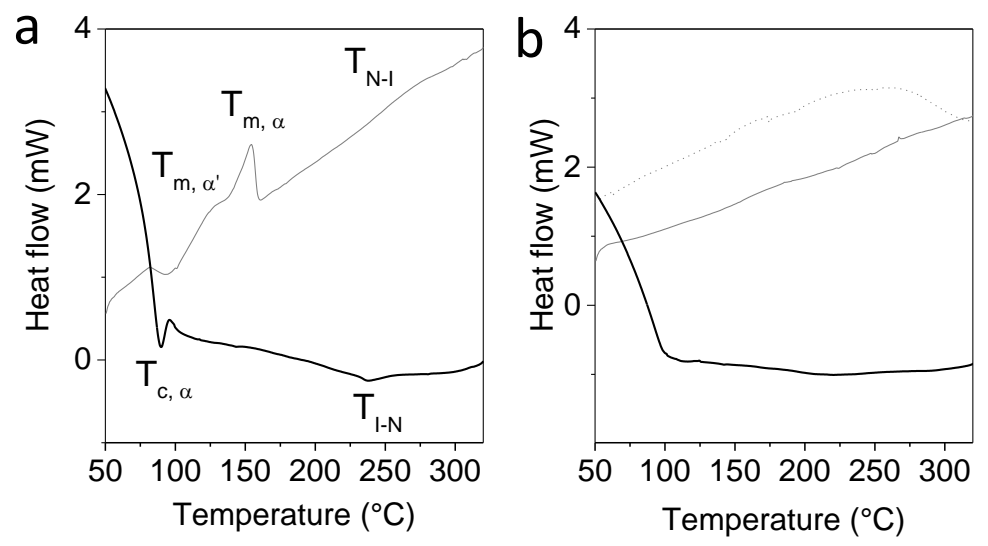

Figure 5. Representative heating (grey lines) and cooling (black lines) DSC traces of bulk PFO (a) and PFO inside $35 \mathrm{~nm}$ in diameter pores (a). Solid line and dotted line in (a) correspond to the heating run of PFO nanowires crystallized in the presence and absence of the surface reservoir, respectively.

DSC experiments were carried out on the PFO confined in $35 \mathrm{~nm}$ pores as well as on the bulk PFO for comparison. Results of the bulk and the nanowires are shown in Figure 5a and 5b, respectively. Although $35 \mathrm{~nm}$ in diameter PFO nanowires were selected due to their high crystallinity (as deduced from WAXS, Figure 3e), just a weak endotherm can be observed in the DSC heating trace (Figure 5a, dotted line), which renders any potential $T_{m}$ analysis difficult. Note that, uncorrected, raw DSC data are presented here in order to provide the reader with an unaltered vision of the behaviour of this system, as any correction of such a weak signal would certainly modify its shape. DSC traces of bulk PFO, in contrast, clearly shows the different thermal processes taking place in the polymer. Thereby, upon heating, one can observe an exothermic process associated with cold crystallization -at $\sim 100{ }^{\circ} \mathrm{C}$, and two overlapped endothermic processes, ascribed to the reorganization of the $\alpha^{\prime}$ phase into the $\alpha$ crystals (at $T_{m, \alpha^{\prime}}$ ) and the subsequent melting of the $\alpha$ crystals into the nematic phase (at $\left.T_{m, \alpha}\right)[25,32]$ 
(Figure $5 b$ grey line). A weak, broad peak ascribed to the nematic to isotropic transition $\left(\mathrm{T}_{\mathrm{N}-\mathrm{I}}\right)$ is also visible at high temperatures. The corresponding isotropic-to-nematic transition $\left(\mathrm{T}_{\mathrm{I}-\mathrm{N}}\right)$ is, however, better appreciated as cooling. Upon further cooling the crystallization of bulk PFO crystals takes place at $\sim 90{ }^{\circ} \mathrm{C}$ (peak temperature).
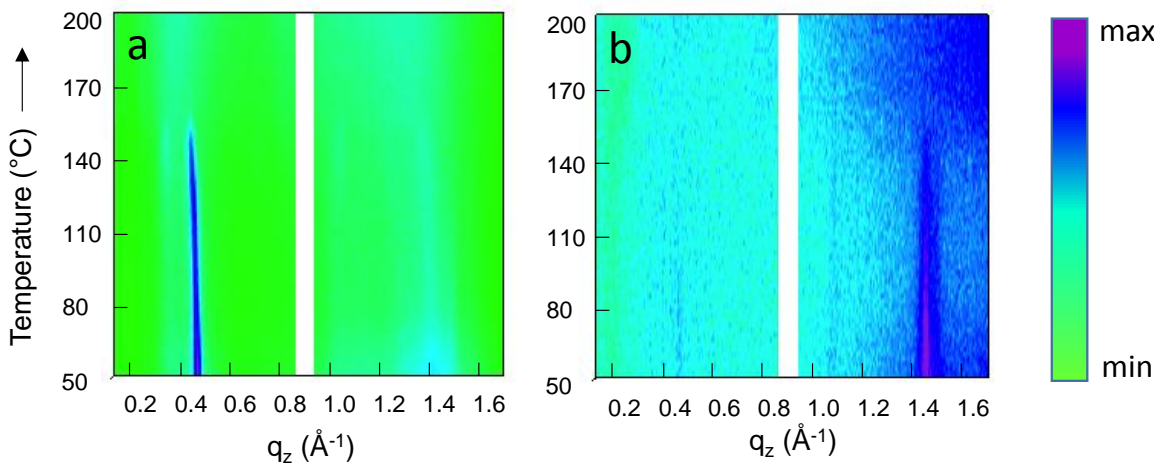

Figure 6. WAXS signal along the $z$-direction upon heating for bulk PFO (a) and $35 \mathrm{~nm}$ in diameter nanowires crystallized at $0.5^{\circ} \mathrm{C} / \mathrm{min}$ with surface reservoir (b). Intensity is plotted as colour scale, the temperature is shown in the vertical direction and $q_{z}$ in the horizontal axis).

Faced with the difficulty of analysing melting temperatures of our confined PFO crystals by DSC, temperature resolved WAXS experiments were performed. In order to compare just the impact of the dimensions of crystals on the $T_{m}$ and thus to avoid effects ascribed to the distinct crystallographic phases, the bulk crystals were transformed into the $\alpha$ phase by thermal annealing at $130{ }^{\circ} \mathrm{C}$ for $10 \mathrm{~min}$, as reported by Chen et al. [25]. Figure 6 depicts the WAXS signal along the $z$-direction as a function of temperature (as heating) and $q_{z}$ for the bulk sample (Figure 6a) and the $35 \mathrm{~nm}$ in diameter nanowires crystallized at $0.5{ }^{\circ} \mathrm{C} / \mathrm{min}$ with surface reservoir (Figure 6b). WAXS intensity is represented in a colour scale, whereas temperature and $q_{z}$ are presented along the vertical and the horizontal axis.

In contrast to the typical behaviour of nanoconfined polymers, the PFO nanowires show similar or even higher $T_{m}\left(T_{m, 35 n m} \sim 151{ }^{\circ} \mathrm{C}\right.$, Figure $\left.6 \mathrm{~b}\right)$ than the bulk counterpart $\left(T_{m, b u l k} \sim 148{ }^{\circ} \mathrm{C}\right.$, Figure 6a). Given that, the only crystal dimension that impacts the melting temperature of bulk crystals is the one along the chain direction, the observed increase of the $T_{m}$ in the nanowires must be correlated to the increase of the lamellar thickness. Moreover, such thickening must be notable because its effect on the $T_{m}$ is probably underestimated in this case. The reason is that whereas the melting point depression of bulk polymers can be just related to the reciprocal 
lamellar thickness $\left(L_{1}^{-1}\right)$ and the free energy of the fold surface $\left(\gamma_{1}\right)$, this simplification is not usually valid for polymer crystals confined in nanopores [1,3]. Given that in our nanowires, the $c$-axis of the crystal cell (the chain direction) points in the direction of the nanopore axis, the other two lateral dimensions of the lamellae lay along the section of nanopores and, therefore, have nanoscale dimensions too. As a consequence, the terms containing the lateral surface energies, $\gamma_{2}$ and $\gamma_{3}$ in equation 1 , are no longer negligible; contributing to the reduction of $T_{m}$. Indeed, notable melting point depressions are measured for polymers confined in AAO nanopores of similar dimension [40,41]. Hence, the $T_{m}$ our confined PFO suggests the development of thicker crystallites, in which the larger size along the chain direction is able to compensate (in fact overcome) the reduction of $T_{m}$ induced by the spatial restriction along the other two spatial dimensions. Note also that the lateral confinement of crystals prevents the employment of the Gibbs-Thomson equation (and the values of $T_{m}^{0}, \Delta H_{f, m}$ and $\gamma_{1}$ reported by Chen and coworkers) to determine the lamellar thickness by this method and prove whether or not extended-chain crystals have been achieved, as the values of the other two interfacial energies are unknown for the PFO[42]. However, it should be pointed out that the formation of thicker crystals might be facilitated in rigid-rod polymers confined in nanopores, as a low density of entanglements can be expected in these systems due to both the nematic nature of the system prior to the crystallization and the typical dilution of the melt entanglement network imposed by the strong spatial confinement [43].

On the other hand, the question why the PFO nanowires slowly crystallized $\left(-0.5^{\circ} \mathrm{C} / \mathrm{min}\right)$ with bulk surface reservoir have a texture which is not governed by typical kinetic selection rules might be related to the large lamellar thickness of the crystals. Note that the lamellar thickness of these crystals seems to be around $23 \mathrm{~nm}$, which directly conflicts with the pore diameter (35 $\mathrm{nm}$ ). In this way, crystals with chains oriented perpendicular to the pore axis (as kinetic selection rules of the crystal growth would dictate) cannot be accommodated in the pore due to space reasons. Thus, the only orientation allowing extended-chain crystals in the pores would be the one in which the chains point in the long axis direction of the pores. The propagation of these crystals could take place through the nucleation of new crystals on the (001) surface of the lamellae, as Wunderlich et al. have demonstrated for extended chain crystals of PE [44]. This would allow the propagation of the crystallization of the nanowires along the $c$-axis direction, and eventually, would explain the anomalous orientation observed in our PFO nanowires. Note also that a surface nucleation process at the AAO pore wall [22], as well as stretched conformation of the chain inside strongly confining pores [21] can also induce the 
observed orientation. However, these possibilities are believed to be unlikely here because both phenomena would occur either if the polymer is crystallized in the presence or absence of surface reservoir. Thus, PFO nanowires crystallized via both crystallization strategies would present equal crystal orientation (c-axis parallel to pore axis), and that is not the case, as will be shown below.

\subsection{Crystallization of the PFO nanowires isolated inside nanopores at high cooling rate}

We analyse now the texture of the PFO nanowires crystallized at $50{ }^{\circ} \mathrm{C} / \mathrm{min}$ while isolated in each nanopore, so that the crystallization occurred independently in each nanopore. Thus, both nucleation and the crystal growth process have to take place under space limitation. To achieve this, samples were quenched in ice-water immediately after the infiltration process and the surface bulk reservoir was removed. The nanowires were then taken to the nematic phase at $200{ }^{\circ} \mathrm{C}$ for $10 \mathrm{~min}$ and crystallized at $50{ }^{\circ} \mathrm{C} / \mathrm{min}$. By using such protocol, we tried to hinder the crystal growth process and, thus, promote a confined crystallization that is dominated by the nucleation process.
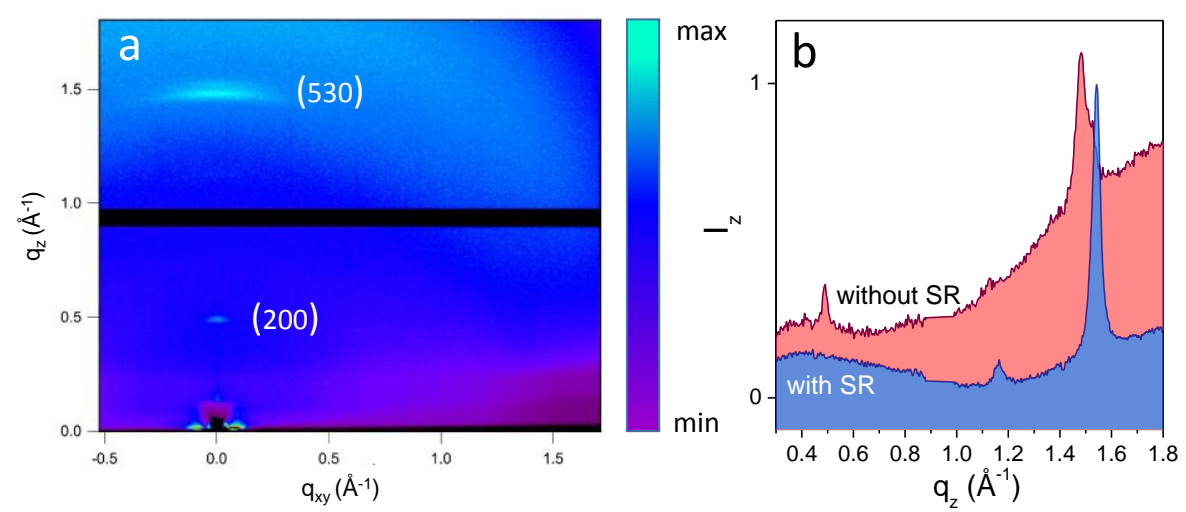

Figure 7. (a) 2D-WAXS patterns of the ensemble of $35 \mathrm{~nm}$ in diameter PFO nanowires crystallized at 50 ${ }^{\circ} \mathrm{C} / \mathrm{min}$ without surface reservoir. (b) WAXS intensity along the $z$-direction (parallel to the long-axis of the nanopores) for $35 \mathrm{~nm}$ in diameter nanowires crystallized in the presence (blue) and in the absence (red) of surface reservoir. Intensity has been normalized to the peak value and then shifted for the clarity of the plot.

The 2D-WAXS patterns are again characterized by well-defined, main discrete reflection at moderately high $q$-values along the meridian, i.e. pore axis direction (Figure 7a). However, a closer view reveals that the texture of these nanowires is substantially different from that of 
nanowires crystallized with the surface reservoir (Figure 7b). The main peak observed is centred at $q=1.48 \AA^{-1}$ ( $d$-spacing of $0.425 \mathrm{~nm}$ ) and can be ascribed to the stacking of (530) lattice planes of the $\alpha$ crystalline phase, with a $d$-spacing of $0.427 \mathrm{~nm}$. A less intense reflection, corresponding to the stacking of (200) lattice planes, can be also observed at $q=0.49 \AA^{-1}$. Thus, the majority of the crystals point their [530] crystallographic direction parallel to the pore axis, although a small crystal fraction exists, in which the [200] direction is aligned to the pore axis. Both crystallographic directions have in common that they are normal to the chain direction in the lattice cell. Therefore, they both correspond to the $[h k l]$ direction with zero $l$-index. Note that nanowires crystallized without the surface reservoir show crystal orientations that differ $90^{\circ}$ from that of the same nanowires crystallized in the presence of the surface reservoir. It is striking that under ideal crystallization conditions for the growth of crystals, i.e. the presence of surface bulk reservoir and a slow cooling rate, $\mathrm{PFO}$ does not show, in principle, a texture governed by kinetics (as the $c$-axis orients parallel to pore axis); whereas, under crystallization conditions that do not promote crystal growth, namely no surface film and high cooling rate $\left(50{ }^{\circ} \mathrm{C} / \mathrm{min}\right)$, the observed texture is the one that one would expect for a kinetically controlled crystallization process.
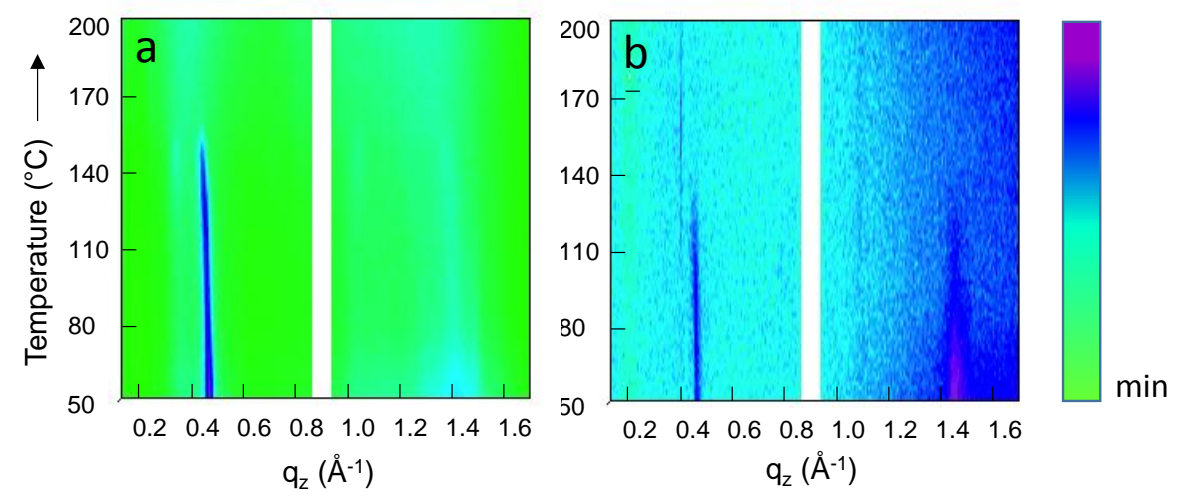

Figure 8. WAXS signal along the $z$-direction upon heating for bulk PFO (a) and crystallized PFO at $50{ }^{\circ} \mathrm{C} / \mathrm{min}$ in pores (35 $\mathrm{nm}$ in diameter) without surface reservoir (b). Intensity is plotted as colour scale, the temperature is shown in the vertical direction and $q_{z}$ in the horizontal axis).

Like in the previous case, temperature resolved WAXS experiments were carried out on these nanowires in order to characterize their thermal behaviour (Figure 8). A significantly lower $T_{m}$ value is measured for the PFO nanowires $\left(T_{m} \sim 129{ }^{\circ} \mathrm{C}\right.$, Figure $\left.8 \mathrm{~b}\right)$ than for bulk PFO $\left(T_{m} \sim\right.$ $148{ }^{\circ} \mathrm{C}$, Figure $8 \mathrm{a}$ ). In the nanowires crystallized without surface film, the $c$-axis of the lattice cell (the chain direction) orients perpendicular to the nanopore long axis, so that one of the lateral dimensions of the lamellae positions normal to the pore axis, while the other lateral 
dimension points along the pore axis. Hence, at least one of the terms containing the lateral surface free energy contribution ( $\gamma_{2}$ in Eq. 1$)$ is not negligible and contributes to the melting point depression. In addition, the formation of thinner lamellae inside pores cannot be ruled out, which would decrease further $T_{m}[20,67]$.

Let us compare now the melting temperature of the nanowires crystallized without surface bulk reservoir $\left(T_{m} \sim 129^{\circ} \mathrm{C}\right)$ with that of the nanowires crystallized in the presence of the surface reservoir $\left(T_{m} \sim 151{ }^{\circ} \mathrm{C}\right)$. We suggest that such difference is basically correlated with the lamellar thickness. As stated in the previous section, nanowires crystallized with surface reservoir are comprised of lamellae that are confined in the three spatial dimensions: the thickness of lamellae $\left(L_{1}\right)$ has always nanoscale size; but, in addition, both lateral dimensions of lamellae $\left(L_{2}\right.$ and $\left.L_{3}\right)$ in these nanowires are also nanoscopic, as these lie in the plane of the pore section. Conversely, in nanowires crystallized without surface reservoir, one of the lateral directions $\left(L_{2}\right)$ is contained in such plane, while $\left(L_{3}\right)$ points along the pore. However, due to the high cooling rate applied for the crystallization of nanowires without surface reservoir (50 ${ }^{\circ} \mathrm{C} / \mathrm{min}$ ), the density of active nucleation centres that initiate crystallization must be very high. Thus, the length of the crystal along $L_{3}$ is likely to be restricted too, in this case by the growthlimited crystallization. Hence, in both kinds of nanowires (i.e. crystallized with and without surface reservoir), crystals are expected to be spatially confined along the three dimensions, and thus the predominant difference in $T_{m}$ is likely to be influenced by the change of lamellar thickness. This assumption is further supported by the fact that the energy of the fold surface $\left(\gamma_{1}\right)$, is typically larger than the lateral surface energies $\left(\gamma_{1}\right.$ and $\left.\gamma_{2}\right)$ in polymer crystals.
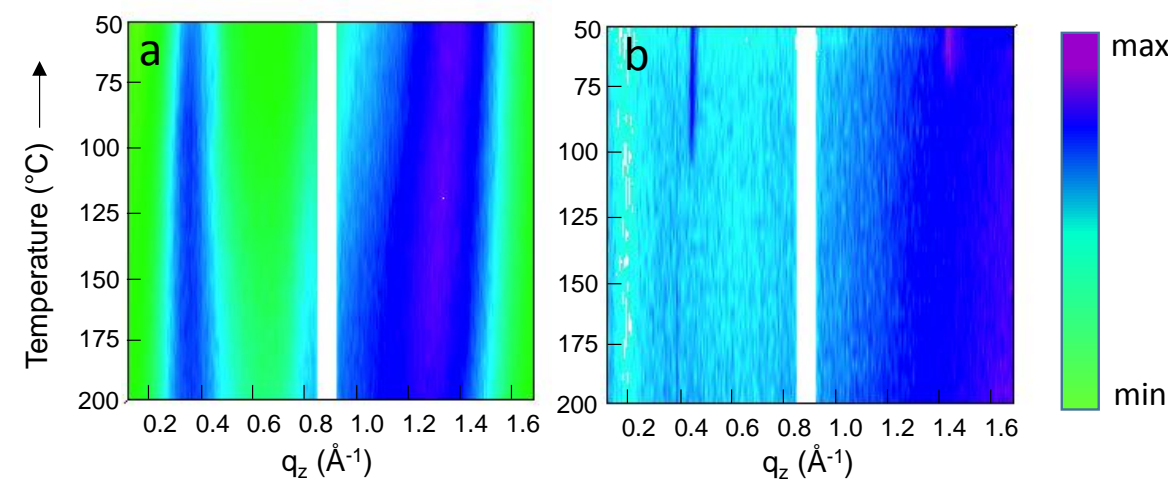

Figure 9. WAXS signal along the $z$-direction upon cooling from $200{ }^{\circ} \mathrm{C}$ for bulk PFO (a) and $35 \mathrm{~nm}$ in diameter nanowires isolated in the pores (no surface reservoir was present on the template) (b) 
Likewise, a similar complex solidification behaviour can be observed upon cooling the nanoconfined PFO from the nematic state (Figure 9b). Indeed, the cooling WAXS colour plot in Figure 9b looks like a mirror image of the one depicting the heating run (Figure 8b). Upon cooling, a diffraction maximum appears in the low $q$ region at $\mathrm{T} \sim 104{ }^{\circ} \mathrm{C}$, which can be related to the intermolecular periodicity along the direction normal to the chain. Upon further cooling, at $\mathrm{T} \sim 66{ }^{\circ} \mathrm{C}$, the peak ascribed to the stacking of (530) lattice planes becomes visible.

Typically employed arguments to justify a two-step crystallization in pores assume either that both heterogeneous and homogeneous nucleation mechanisms are active, or that secondary nucleation processes are detectable. Nevertheless, the fact that the first noticeable peak is the one corresponding to the interchain correlation -represented by the (200) reflection- leads us to consider also another possibility: the development of a non-crystalline, yet well-ordered structure at $\mathrm{T} \sim 104{ }^{\circ} \mathrm{C}$, i.e. the $\alpha^{\prime}$ mesophase mentioned before when crystallization is dominated by the nucleation process. This structure would be characterized by a well-defined periodicity along the $a$-axis (by analogy with the crystal cell) but absence of long-range order in other directions. Then, upon further cooling, such structure would evolve to well-defined $\alpha$ crystals that would be fully visible at $\mathrm{T} \sim 66^{\circ} \mathrm{C}$. This intermediate structure would act as a bridge between the liquid nematic phase and the $\alpha$ crystal and thus it would also transfer the molecular orientation from the nematic to the crystal form. In this way, the rigid PFO chains are probably already oriented perpendicular to pore axis in the nematic mesophase prior to the nucleation of the $\alpha^{\prime}$ entities, and the molecular orientation is preserved during crystallization, so that, eventually, crystallographic directions perpendicular to the chain axis, i.e. [530] and [200], become positioned parallel to the pore axis. Note that, this hypothesis implies that the orientation observed in PFO nanowires crystallized without surface reservoir does not have a kinetic origin either, even though only $[h k 0]$ directions are visible along the pore axis, which would resolve the paradox of why nanowires crystallized slowly with surface film did not show a texture governed by kinetics, while fast crystallization without surface film seemed to induce kinetically controlled orientations. In fact, when low cooling rates are applied on the samples not containing the surface reservoir, the [008] crystallographic direction positions preferentially along to the pore long axis (see Suplementary Information, Fig. S1.). This means that when confined PFO crystals are allowed to propagate over a certain distance, crystals probably grow preferentially along the [008] direction. Thus, orientations with $[h k 0]$ directions parallel to pore axis would be consequence of a nucleation dominated crystallization. 


\section{Conclusions}

We have demonstrated that the development of the internal structure of the rigid-rod, semiconducting polymer PFO can be effectively controlled by the 2D spatial confinement and the employment of crystallization strategies. The effect of nanoconfinement on the overall crystallization process depends strongly on the individual relationship between the crystal nucleation and the crystal growth with the available space. Hence, the application of crystallization strategies that impact the nucleation and growth processes allows for the manipulation of the crystal texture of conjugated polymers in confinement and, thus, to produce semiconducting nanowires with tailored microstructures.

When PFO is crystallized inside the nanopores in the presence of a bulk surface reservoir highly crystalline and textured nanowires are achieved, in which crystals are uniaxially oriented pointing the chain direction in the crystal, i.e. the $c$-axis, parallel to the long axis of the nanopores. Interestingly, the large value of coherence length of the $(00 l)$ reflection along the pore direction and the slight increase of the melting temperature of these nanowires as compared to bulk PFO crystals suggest the formation of thicker PFO lamellae inside the nanopores. The possibility that extended-chain crystals of polymeric semiconductors would be forming within AAO nanopores is still an open question that would be worth resolving, as it is well known that optoelectronic properties of many high-performing, rigid-rod semiconducting polymers, such as poly(2,5-bis(3-alkylthiophen-2-yl)thieno[3,2-b]thiophenes) (PBTTT)[68], or diketopyrrolopyrrole (DPP) based polymers [69], are directly correlated with their packing and internal microstructure.

Conversely, PFO nanowires crystallized isolated inside the nanopores (without the surface reservoir) exhibit a preferential texture in which chains are oriented normal to the long axis of the pores. That is, the crystal orientation in these nanowires differs $90^{\circ}$ from that of the same nanowires crystallized in the presence of the surface reservoir. We propose that the multiple nucleation events that must occur inside the pores due to the isolated crystallization and fast cooling process provoke a growth-limited crystallization in which the molecular orientation is transferred from the nematic phase to the $\alpha^{\prime}$ solid-state mesophase and, eventually, to the $\alpha$ crystals.

The findings presented here provide an excellent example of the simple fabrication and structural manipulation of ordered arrays of well-aligned semiconducting nanowires that can be achieved via confined crystallization, which may help establishing the foundation of a new 
range of architectures/devices with highly-anisotropic-response, such as polarized electroluminescence devices, waveguides, optical switching devices, lasers, and more.

\section{Acknowledgements}

Jaime Martín acknowledges support from the European Union's Horizon 2020 research and innovation programme under the Marie Skłodowska-Curie grant, agreement No 654682.The work has been partially supported under a KAUST Global Collaborative Research Academic Excellence Alliance (AEA) grant. This work is based upon research conducted at the Cornell High Energy Synchrotron Source (CHESS) which is supported by the National Science Foundation and the National Institutes of Health/National Institute of General Medical Sciences under NSF award DMR-1332208.

\section{References}

[1] Alcoutlabi M, McKenna G, B. Effects of confinement on material behaviour at the nanometre size scale. J. Phys.: Condens. Matter. 2005;17(15):R461.

[2] Huber P. Soft matter in hard confinement: phase transition thermodynamics, structure, texture, diffusion and flow in nanoporous media. J. Phys.: Condens. Matter. 2015;27(10):103102.

[3] Jiang Q, Ward MD. Crystallization under nanoscale confinement. Chem. Soc. Rev. 2014;43(7):2066-2079.

[4] O'Carroll D, Lieberwirth I, Redmond G. Microcavity effects and optically pumped lasing in single conjugated polymer nanowires. Nat. Nanotechnol. 2007;2(3):180-184.

[5] Grimm S, Martin J, Rodriguez G, Fernandez-Gutierrez M, Mathwig K, Wehrspohn RB, et al. Cellular interactions of biodegradable nanorod arrays prepared by nondestructive extraction from nanoporous alumina. J. Mater. Chem. 2010;20(16):3171-3177.

[6] Heeger AJ. 25th Anniversary Article: Bulk Heterojunction Solar Cells: Understanding the Mechanism of Operation. Adv. Mater. 2014;26(1):10-28.

[7] Friend RH, Gymer RW, Holmes AB, Burroughes JH, Marks RN, Taliani C, et al. Electroluminescence in conjugated polymers. Nature. 1999;397(6715):121-128.

[8] Noriega R, Rivnay J, Vandewal K, Koch FPV, Stingelin N, Smith P, et al. A general relationship between disorder, aggregation and charge transport in conjugated polymers. Nat. Mater. 2013;12(11):1038-1044.

[9] Michell RM, Blaszczyk-Lezak I, Mijangos C, Müller AJ. Confinement effects on polymer crystallization: From droplets to alumina nanopores. Polymer. 2013;54(16):4059-4077.

[10] Michell RM, Blaszczyk-Lezak I, Mijangos C, Müller AJ. Confined crystallization of polymers within anodic aluminum oxide templates. J. Polym. Sci. B: Polym. Phys. 2014;52(18):1179-1194.

[11] Martín J, Nogales A, Mijangos C. Directional Crystallization of $20 \mathrm{~nm}$ Width Polymer Nanorods by the Inducement of Heterogeneous Nuclei at Their Tips. Macromolecules. 2013;46(18):7415-7422.

[12] Woo E, Huh J, Jeong YG, Shin K. From Homogeneous to Heterogeneous Nucleation of Chain Molecules under Nanoscopic Cylindrical Confinement. Phys. Rev. Lett. 2007;98(13):4. 
[13] Masuda H, Fukuda K. Ordered Metal Nanohole Arrays Made by a Two-Step Replication of Honeycomb Structures of Anodic Alumina. Science. 1995;268(5216):1466-1468.

[14] Martín J, Manzano CV, Caballero-Calero O, Martín-González M. High-Aspect-Ratio and Highly Ordered 15-nm Porous Alumina Templates. ACS Appl. Materi. Interfaces. 2013;5(1):72-79.

[15] Aryal M, Trivedi K, Hu W. Nano-Confinement Induced Chain Alignment in Ordered P3HT Nanostructures Defined by Nanoimprint Lithography. ACS Nano. 2009;3(10):3085-3090.

[16] Byun J, Kim Y, Jeon G, Kim JK. Ultrahigh Density Array of Free-Standing Poly(3hexylthiophene) Nanotubes on Conducting Substrates via Solution Wetting. Macromolecules. 2011;44(21):8558-8562.

[17] Kim JS, Park Y, Lee DY, Lee JH, Park JH, Kim JK, et al. Poly(3-hexylthiophene) Nanorods with Aligned Chain Orientation for Organic Photovoltaics. Adv. Funct. Mater. 2010;20(4):540-545.

[18] Santos A, Formentín P, Pallarés J, Ferré-Borrull J, Marsal LF. Fabrication and characterization of high-density arrays of P3HT nanopillars on ITO/glass substrates. Solar Energy Materials \& Solar Cells. 2010;94(7):1247-1253.

[19] Martin J, Campoy-Quiles M, Nogales A, Garriga M, Alonso MI, Goni AR, et al. Poly(3hexylthiophene) nanowires in porous alumina: internal structure under confinement. Soft Matter. 2014;10(18):3335-3346.

[20] O' Carroll D, Irwin J, Tanner DA, Redmond G. Polyfluorene nanowires with pronounced axial texturing prepared by melt-assisted template wetting. Mater. Sci. Eng., B 2008;147(2-3):298-302.

[21] Guan Y, Liu G, Gao P, Li L, Ding G, Wang D. Manipulating Crystal Orientation of Poly(ethylene oxide) by Nanopores. ACS Macro Letters. 2013;2(3):181-184.

[22] Wu H, Wang W, Huang Y, Su Z. Orientation of Syndiotactic Polystyrene Crystallized in Cylindrical Nanopores. Macromol. Rapid Commun.. 2009;30(3):194-198.

[23] O'Carroll D, Lieberwirth I, Redmond G. Melt-Processed Polyfluorene Nanowires as Active Waveguides. Small. 2007;3(7):1178-1183.

[24] Grell M, Bradley DDC, Inbasekaran M, Woo EP. A glass-forming conjugated main-chain liquid crystal polymer for polarized electroluminescence applications. Adv. Mater. 1997;9(10):798-802.

[25] Chen SH, Su AC, Su CH, Chen SA. Crystalline Forms and Emission Behavior of Poly(9,9-di-noctyl-2,7-fluorene). Macromolecules. 2005;38(2):379-385.

[26] Grell M, Bradley DDC, Long X, Chamberlain T, Inbasekaran M, Woo EP, et al. Chain geometry, solution aggregation and enhanced dichroism in the liquidcrystalline conjugated polymer poly $(9,9-$ dioctylfluorene). Acta Polym. 1998;49(8):439-444.

[27] Martín J, Nogales A, Martín-González M. The Smectic-Isotropic Transition of P3HT Determines the Formation of Nanowires or Nanotubes into Porous Templates. Macromolecules. 2013;46(4):14771483.

[28] Auriemma F, De Rosa C, Corradini P. Solid Mesophases in Semicrystalline Polymers: Structural Analysis by DiffractionTechniques. In: Allegra G, editor. Interphases and Mesophases in Polymer Crystallization II: Springer Berlin Heidelberg; 2005. p. 1-74.

[29] Bonart R. Kolloid Z Z Polym. 1966;213.

[30] de Candia F, Filho AR, Vittoria V. Strain-induced mesomorphic order in glassy syndiotactic polystyrene. Makromol. Chem., Rapid Commun.. 1991;12(5):295-299.

[31] Vittoria, Guadagno L, Comotti A, Simonutti R, Auriemma F, De Rosa C. Mesomorphic Form of Syndiotactic Polypropylene. Macromolecules. 2000;33(16):6200-6204. 
[32] Chen SH, Su AC, Chen SA. Noncrystalline Phases in Poly(9,9-di-n-octyl-2,7-fluorene). J. Phys. Chem. B. 2005;109(20):10067-10072.

[33] Grell M, Bradley DDC, Ungar G, Hill J, Whitehead KS. Interplay of Physical Structure and Photophysics for a Liquid Crystalline Polyfluorene. Macromolecules. 1999;32(18):5810-5817.

[34] Chen SH, Chou HL, Su AC, Chen SA. Molecular Packing in Crystalline Poly(9,9-di-n-octyl-2,7fluorene). Macromolecules. 2004;37(18):6833-6838.

[35] Steinhart M, Goring P, Dernaika H, Prabhukaran M, Gosele U, Hempel E, et al. Coherent Kinetic Control over Crystal Orientation in Macroscopic Ensembles of Polymer Nanorods and Nanotubes. Phys. Rev. Lett.. 2006;97(2):027801.

[36] Lim JA, Liu F, Ferdous S, Muthukumar M, Briseno AL. Polymer simiconductor crystals. Materials Today. 2010;13(5).

[37] Scherrer P. Nachr Ges Wiss Gottingen Math-Phys Klasse. 1918(26):2.

[38] Gibbs JW. Collected Works. New York: Yale University Press; 1928.

[39] Hoffman JD, Frolen LJ, Ross GS, Lauritzen JI. On the growth rate of spherulites and axialites from the melt in polyethylene fractions: Regime I and regime II crystallization. J Res Natl Bur Stand Sect A: Phys Chem. 1975;79.

[40] Choi K, Lee SC, Liang Y, Kim KJ, Lee HS. Transition from Nanorod to Nanotube of Poly(vinylidene trifluoroethylene) Ferroelectric Nanofiber. Macromolecules. 2013;46(8):3067-3073.

[41] Shin K, Woo E, Jeong YG, Kim C, Huh J, Kim K-W. Crystalline Structures, Melting, and Crystallization of Linear Polyethylene in Cylindrical Nanopores. Macromolecules. 2007;40(18):66176623.

[42] Chen SH, Su CH, Su AC, Sun YS, Jeng U, Chen SA. Gibbs-Thomson analysis of crystalline poly(9,9-di-n-octyl-2,7-fluorene). J. Appl. Crystall.. 2007;40(s1).

[43] Martín J, Krutyeva M, Monkenbusch M, Arbe A, Allgaier J, Radulescu A, et al. Direct Observation of Confined Single Chain Dynamics by Neutron Scattering. Phys. Rev. Lett.. 2010;104(19):197801.

[44] Wunderlich B, Mielillo L. Morphology and growth of extended chain crystals of polyethylene. Makromol. Chem. 1968;118(1):250-264. 